\title{
Resultados Neonatais no Parto Vaginal Espontâneo Comparados aos dos Partos com Fórcipe de Simpson-Braun em Primíparas
}

\author{
Neonatal Outcome of Spontaneous Deliveries as Compared to \\ Simpson-Braun Forceps Deliveries in Nulliparous Women \\ Belmiro Gonçalves Pereira, Michel Gardere Camargo, Egle Cristina Couto, \\ Eliana Amaral, Renato Passini Jr, Mary Angela Parpinelli
}

\begin{abstract}
RESUMO
Objetivo: comparar os resultados neonatais dos partos vaginais espontâneos ou assistidos com fórcipe de Simpson-Braun em nulíparas.

Método: em estudo de corte retrospectivo foram avaliados dois grupos de primíparas atendidas no Centro Obstétrico do CAISM/UNICAMP, que tiveram parto vaginal sob analgesia epidural. O grupo fórcipe foi formado por 119 pacientes que tiveram parto a fórcipe de Simpson-Braun, e o grupo normal por 114 casos de parto vaginal espontâneo. Foram estudadas as variáveis neonatais imediatas como o estado do líquido amniótico e os índices de Apgar, assim como a evolução neonatal nos primeiros dias de vida. Para análise estatística foram utilizados os testes $\chi^{2}$, exato de Fisher e $\mathrm{t}$ de Student para comparação de médias e considerada significativa a diferença correspondente a $p<0,05$.

Resultados: as indicações para uso do fórcipe Simpson-Braun foram: alivio materno-fetal (90 casos) e abreviação de periodo expulsivo (29 casos). Nas pacientes atendidas com fórcipe Simpson-Braun houve 8 casos de laceração de canal de parto (6,7\%), valor semelhante ao observado no grupo de parto vaginal espontâneo. As médias de dias de internação das puérperas e neonatos foram idênticas $(2,4)$. Os dois grupos foram semelhantes quanto aos indices de Apgar menores que 7 no primeiro (7,5 e 4,3\%) e quinto minuto (1,6 e 1,7\%) $e$ quanto ao peso dos recém-nascidos (3.146 e $3.016 \mathrm{~g})$. A evolução neonatal foi semelhante nos dois grupos.

Conclusão: o uso do fórcipe de Simpson-Braun mostrou-se seguro, quando comparado ao parto vaginal espontâneo.
\end{abstract}

PALAVRAS-CHAVE: Parto. Fórcipe. Morte materna. Resultados neonatais.

\section{Introdução}

O resgate do parto vaginal é tendência universalmente aceita. Parece haver consenso sobre as vantagens do parto vaginal em relação à

Centro de Atenção Integral à Saúde da Mulher (CAISM) Setor de Obstetrícia do Departamento de Tocoginecologia da Faculdade de Ciências Médicas da Universidade Estadual de Campinas

Endereço:

Rua Alexander Fleming 101

Cidade Universitária "Zeferino Vaz"

13084-881 - Campinas - SP

Fone: (19) 3788-9402/Fax (19) 3289-5935

e-mail: belmirop@hotmail.com

Financiamento: Auxílio-pesquisa de iniciação científica do CNPq cesárea, que tem atingido niveis alarmantes nas últimas décadas. $O$ conhecimento acerca das formas de resolução espontânea ou instrumental do parto vaginal tem merecido especial atenção, particularmente o uso do fórcipe.

O fórcipe de Simpson-Braun é instrumento destinado a extrair fetos por preensão de seu pólo cefálico $^{1}$. Trata-se de instrumento médico utilizado desde a Antigüidade. Entretanto, seu uso é polêmico principalmente pelo fato de sua utilização ter sido mais difundida e aplicada numa fase da Obstetrícia na qual o que importava era a vida materna, podendo o feto ser sacrificado.

Quando bem utilizado, no entanto, é instrumento valioso, colaborando de forma efetiva e im- 
portante para o parto vaginal ${ }^{2}$. Infelizmente, o estigma que lhe foi imputado pelas antigas práticas obstétricas persiste entre os leigos e entre aqueles que não foram adequadamente treinados para seu uso.

Atualmente, não se aplica o fórcipe em cabeças altas ou encravadas no estreito médio do canal de parto (contra-indicação absoluta) ${ }^{2}$. O fórcipe tem comprovada eficácia em evitar a hipóxia fetal, abreviar o período expulsivo e minimizar os riscos de seqüelas neurológicas ${ }^{2}$.

A utilização do fórcipe torna-se justificável em situações que ameaçam o bem-estar materno e/ou fetal e que podem ser revertidas pelo uso adequado e seguro do instrumento. Algumas indicações maternas incluem cardiopatias e pneumopatias graves e eclâmpsia, dentre outras. As indicações fetais incluem sofrimento fetal agudo, pequenas distocias de rotação, alivio maternofetal e abreviação do período expulsivo ${ }^{2}$.

Conhecer os benefícios e efeitos adversos do fórcipe de Simpson-Braun representa importante tarefa, particularmente a avaliação dos resultados perinatais. Na literatura existem poucos dados consistentes, com estudos controlados, sobre a aplicação do fórcipe.

Em estudo de metanálise comparando o fórcipe com o vácuo-extrator, por exemplo, evidenciou-se que este último está associado a menor morbidade materna, reduzindo traumas, dor perineal, o uso de anestesias geral e regional, assim como redução dos índices de cesárea. O fórcipe, entretanto, esteve menos associado a céfalo-hematomas e hemorragias retinianas nos neonatos. Vale destacar, no entanto, que os recém-nascidos não apresentaram danos sérios com nenhum dos instrumentos ${ }^{3}$.

Um estudo de 130 casos, ao longo de 15 anos, mostrou resultados excelentes e concluiu que o fórcipe é alternativa segura para a finalização do parto ${ }^{4}$. Ezenagu et al. ${ }^{5}$ acompanharam 34 parturientes por fórcipe e constataram que seu uso prudente e adequado não contribui para aumentos nas taxas de morbidade materna ou neonatal. Johanson et al. ${ }^{6}$ acompanharam 115 parturientes e recém-nascidos por fórcipe durante 5 anos após o parto, e não encontraram evidências para afirmar que este instrumento traga malefícios à mãe ou à criança.

Entretanto, MacArthur et al. ${ }^{7}$ apresentaram o fórcipe como um fator de risco para a incontinência fecal.

O objetivo deste estudo foi avaliar e comparar alguns resultados maternos e neonatais nos partos vaginais espontâneos e nos partos vaginais assistidos por fórcipe de Simpson-Braun.

\section{Pacientes e Métodos}

Trata-se de estudo retrospectivo de corte transversal realizado no Centro de Atenção Integral à Saúde da Mulher (CAISM) da Universidade Estadual de Campinas (UNICAMP). Este estudo foi aprovado pela Comissão de Pesquisa do Departamento de Tocoginecologia e pelo Comitê de Ética em Pesquisa da Faculdade de Ciências Médicas da UNICAMP, obtendo financiamento do CNPq.

Foram analisados 119 partos vaginais de primíparas atendidas com fórcipe Simpson-Braun (grupo fórcipe) e sob condições de analgesia, no período de 1993 a 2000. Os resultados maternos e neonatais foram comparados aos de 114 partos vaginais espontâneos também de primiparas, realizados no mesmo período e nas mesmas condições de analgesia, formando um grupo-controle (grupo normal). O objetivo foi avaliar e comparar os resultados maternos e neonatais nos partos vaginais espontâneos e nos partos vaginais assistidos por fórcipe de Simpson-Braun, em primíparas, realizados neste período.

Os critérios de inclusão utilizados foram: gestação com mais de 30 semanas de feto único em apresentação cefálica, feto vivo na admissão até o período expulsivo e analgesia de parto. Os critérios de exclusão foram: fichas incompletamente preenchidas, fetos malformados e fetos macrossômicos (>4.000 gramas). Os dois grupos mostraram-se comparáveis de acordo com as variáveis de controle: idade materna, acompanhamento pré-natal e idade gestacional por ocasião do parto (v. Tabela 1).

Foram comparadas as complicações maternas (lacerações, hemorragia e infecção puerperal) e as condições de alta. Da mesma forma, foram comparadas as condições neonatais imediatas: estado do líqüido amniótico, classificado como claro ou tinto (normal), espesso ou antigo $(3+/ 4+)$, índice de Apgar e as condições e complicações neonatais nos primeiros dias de evolução (hemorragias extra e/ou intracranianas, marcas cutâneas da "pega" do fórcipe, equimoses e lesões neurológicas), assim como as condições de alta (sadio ou com seqüelas).

Os dados foram obtidos de um banco de dados, formado a partir de informações contidas em ficha pré-codificada (ficha obstétrica - FO) do Setor de Obstetrícia do CAISM. Quando os dados foram conflitantes ou incompreensiveis por meio dessa ficha, foram revisados os prontuários clínicos das pacientes. Os dados foram inseridos e analisados no programa Epi-Info 6.04, sendo digitados por dois operadores em duas ocasiões diferentes 
para serem submetidos a testes de consistência e correção. Os dados em ambos os grupos foram comparados e para a análise estatística foi utilizado o teste $\chi^{2}$ simples, e quando as freqüências observadas foram baixas usou-se a correção de Yates ou o teste exato de Fisher, e para comparação de médias o teste $t$ de Student. Foi considerada estatisticamente significativa a diferença correspondente a $\mathrm{p}<0,05$.

\section{Resultados}

Em três quartos das pacientes atendidas com fórcipe, este foi indicado para alivio maternofetal (90 casos) e os restantes para abreviação de período expulsivo (29 casos).

Os dois grupos (fórcipe e normal) eram comparáveis quanto à história obstétrica, idade materna, freqüência do pré-natal, número de consultas durante o pré-natal e idade gestacional referida (Tabela 1). Quatro pacientes no grupo de parto vaginal espontâneo e duas no grupo de fórcipe não receberam assistência pré-natal. Seis gestantes no grupo atendido com parto vaginal espontâneo e cinco no grupo atendido com fórcipe tinham antecedentes de aborto. Houve um caso de parto prematuro no grupo fórcipe Simpson-Braun $(0,84 \%)$ e quatro casos no grupo de parto vaginal espontâneo $(3,5 \%)$, não havendo diferença estatisticamente significativa.

Tabela 1 - Algumas características das pacientes dos dois grupos de estudo.

\begin{tabular}{lccccc}
\hline & \multicolumn{3}{c}{ Grupo fórcipe } & \multicolumn{4}{c}{ Grupo normal } \\
Variável & Média & DP & Média & $\mathbf{5 , 2}$ & $\mathbf{p}$ \\
\hline Idade materna & 20,9 & 5,5 & 20,6 & & NS* \\
Número de consultas & 7,1 & 2,7 & 6,2 & 5,9 & NS* $^{*}$ \\
Idade gestacional & 39,5 & 2,1 & 39,0 & 2,0 & NS*
\end{tabular}

$\mathrm{DP}=$ desvio-padrão; $\mathrm{p}=$ significância estatística; NS = não significativo; test $t$ de Student

Entre as mulheres atendidas com fórcipe Simpson-Braun houve 8 casos de laceração do canal de parto $(6,7 \%)$, valor semelhante ao observado no grupo de parto vaginal espontâneo $(6 \mathrm{ca}-$ sos $=5,5 \%$ ). As médias de dias de internação das puérperas e neonatos foram idênticas: 2,4 dias. Nove parturientes do grupo de parto fórcipe e cinco do grupo espontâneo necessitaram de quatro ou mais dias de internação.

Houve um caso de hemorragia puerperal em uma gestante atendida com parto vaginal espontâneo. No grupo de parto fórcipe houve um caso de inversão uterina total e hemorragia (pré-choque) durante o parto, seguida de infecção puerperal. Houve um caso de óbito materno, ocorrido no puerpério tardio, em uma puérpera cardiopata atendida por parto vaginal espontâneo. A paciente era portadora de defeito do septo atrial, que evoluiu com arritmia supraventricular, hipertensão pulmonar e insuficiência cardíaca. Dezessete pacientes do grupo fórcipe (14\%) e oito no grupo normal (7\%) apresentavam alguma doença associada à gestação.

Em relação aos índices de Apgar menores que 7 no primeiro minuto, observamos na Tabela 2 que foram semelhantes no grupo atendido com fórcipe de Simpson-Braun e no grupo atendido com parto vaginal espontâneo $(7,5$ e $4,3 \%$, respectivamente). Da mesma forma, os índices de Apgar de quinto minuto menores que 7 foram similares nos grupos fórcipe de Simpson-Braun e vaginal espontâneo (1,6 e 1,7\%). Também não apareceram diferenças estatisticamente significativas entre os dois grupos quanto à ocorrência de mecônio $(10,9$ e 14,0\%) (Tabela 2).

Tabela 2 - Apgar de $1^{\circ}$ e $5^{\circ}$ minuto e presença de mecônio no líquido amniótico em recém-nascidos dos dois grupos estudados.

\begin{tabular}{|c|c|c|c|c|c|c|c|}
\hline \multirow[t]{2}{*}{ Variável } & \multicolumn{2}{|c|}{$\begin{array}{l}\text { Grupo fórcipe } \\
\qquad(n=119)\end{array}$} & \multicolumn{2}{|c|}{$\begin{array}{c}\text { Grupo (AMF) } \\
(n=90)\end{array}$} & \multicolumn{2}{|c|}{$\begin{array}{l}\text { Grupo norma } \\
\qquad(n=114)\end{array}$} & \multirow{2}{*}{$\begin{array}{l}\text { p } \\
\% \\
\end{array}$} \\
\hline & $n$ & $\%$ & $\mathrm{n}$ & $\%$ & $\mathrm{n}$ & $\%$ & \\
\hline Apgar $1^{\circ}$ minuto <7 & 9 & 7,5 & 4 & 4,4 & 5 & 4,3 & NS \\
\hline Apgar $5^{\circ}$ minuto $<7$ & 2 & 1,6 & 0 & 0 & 2 & 1,7 & NS \\
\hline Mecônio (3+/4+) & 13 & 10,9 & 10 & 11,1 & 16 & 14,0 & NS \\
\hline $\begin{array}{l}\text { *Teste } \chi^{2} \text {. } \\
\text { NS = não significativo. } \\
n=\text { número de casos. } \\
\text { AMF = alívio materno feta } \\
p=\text { significância estatísti }\end{array}$ & & & & & & & \\
\hline
\end{tabular}

Quanto à indicação do uso de fórcipe, cerca de dois terços foi indicado para alívio materno fetal e os demais para abreviação do período expulsivo (29 casos). Entre os casos com a segunda indicação foi encontrada maior freqüência de índice de Apgar de primeiro minuto menor que 7, com diferença significativa $(\mathrm{p}<0,05)$. Da mesma forma, os índices de Apgar de quinto minuto menores que 7 foram mais freqüentes neste grupo, porém sem diferença estatisticamente significativa.

Em relação à idade gestacional somática (Capurro) dos recém-nascidos dos dois grupos houve semelhança, com média de 39,6 semanas no grupo fórcipe e 39,3 semanas no grupo normal (Tabela 3). Em relação ao peso dos recém-nascidos dos dois grupos houve semelhança, com média de 3.146 gramas no grupo fórcipe e 3.016 gramas no grupo normal. Notamos que o peso dos neonatos do grupo fórcipe foi maior que os do grupo normal, mas sem diferença estatisticamente significati- 
va (Tabela 3). Quando os partos a fórcipe foram divididos pela indicação em alívio materno-fetal e abreviação do período expulsivo, os recém-nascidos de partos com a segunda indicação apresentaram maior média de peso (3.230 gramas) do que o grupo de alívio (3.118 gramas), sem diferença significativa. Entretanto, a média de peso dos neonatos atendidos com fórcipe para abreviação do período expulsivo (3.230 gramas) foi maior que a do grupo normal (3.016 gramas), com diferença significativa $(\mathrm{p}<0,05)$.

Tabela 3 - Peso e idade gestacional somática (Capurro) dos recém-nascidos nos dois grupos estudados.

\begin{tabular}{lccccc}
\hline \multirow{2}{*}{ Variável } & \multicolumn{2}{c}{ Grupo fórcipe } & \multicolumn{3}{c}{ Grupo normal } \\
& Média & DP & Média & DP & $\mathbf{p}^{*}$ \\
\hline Peso em gramas & 3.145 & 400,0 & 3.016 & 441,5 & NS \\
Capurro em semanas & 39,6 & 1,2 & 39,3 & 2,8 & NS \\
\hline
\end{tabular}

*Teste $t$ de Student

NS = não significativo

No grupo normal houve um caso de óbito de neonato prematuro, algumas semanas após o parto por complicações infecciosas e respiratórias. A criança nasceu com Capurro de 32 semanas e 4 dias, peso de 1.130 gramas e Apgar 1/6. O exame da placenta mostrou corioamnionite aguda moderada e hematoma subcoriônico.

No grupo de parto a fórcipe para abreviação do período expulsivo houve um caso de anóxia neonatal grave. Durante o parto, o feto apresentou-se com circular justa de cordão e distocia de ombro, havendo rotura de cordão. O neonato apresentava Capurro de 40 semanas, peso de 3.630 gramas e Apgar 1/2. O neonato permaneceu internado na UTI neonatal por 10 dias e teve alta com seqüelas graves.

\section{Discussão}

Do ponto de vista metodológico, os dois grupos eram comparáveis, pois as variáveis idade materna, assistência pré-natal, idade gestacional somática (Capurro) e estado do líquido amniótico foram similares. Em ambos os grupos, as parturientes estavam sob analgesia de condução. Portanto, alguns possiveis vieses foram controlados.

De um modo geral, os resultados maternos em partos de primíparas atendidas com parto fórcipe de Simpson-Braun, para alívio maternofetal ou abreviação do período expulsivo, não foram diferentes daqueles encontrados nas pacientes primíparas atendidas com parto vaginal espontâneo. Em nosso estudo, a incidência de laceração do canal de parto foi semelhante nos dois grupos. Robinson et al. ${ }^{8}$, ao analisarem 323 partos vaginais consecutivos, concluíram que o fórcipe não é fator de risco para a laceração do canal de parto. Em outro estudo, Wood et al. ${ }^{9}$, apontaram o fórcipe como fator de risco para lesão do esfíncter anal, que não observamos neste estudo.

Entretanto, quando se analisam os resultados neonatais verifica-se que houve maior freqüência de índices de Apgar de primeiro minuto menores que 7 nos partos a fórcipe para abreviação do período expulsivo quando comparados aos partos vaginais espontâneos e fórcipe para alívio materno-fetal.

Quanto aos índices de Apgar de quinto minuto menores que 7 , observamos que não houve diferença entre os dois grupos: fórcipe ou parto espontâneo. Entretanto, quando os partos fórcipe para foram divididos em alívio materno-fetal e abreviação de período expulsivo, este apresenta maior freqüência de casos, embora sem diferença significativa. Os grupos atendidos com fórcipe alivio materno-fetal e parto vaginal espontâneo foram similares quanto à freqüência de índices de Apgar de primeiro e quinto minutos.

É possível que o maior peso dos neonatos no grupo fórcipe para abreviação do período expulsivo seja responsável pelo maior tempo de período expulsivo, sendo necessária a indicação de fórcipe. Com o maior tempo de período expulsivo, poderiam ser explicados os menores índices de Apgar de primeiro e quinto minuto dos neonatos desse grupo.

Embora se atribua ao fórcipe um aumento na ocorrência de lesão intracraniana, Towner et al. ${ }^{10}$ observaram que o tipo de parto não é fator de risco para este tipo de lesão em nulíparas. Em nosso estudo não observamos lesão intracraniana.

Em concordância com nosso estudo, Carmona et al. ${ }^{11}$ concluíram que o fórcipe baixo eletivo pode ser usado para encurtar o segundo estágio do parto, sem que haja efeitos colaterais maternos ou neonatais imediatos. Wydra et al. ${ }^{12}$ compararam 170 partos a fórcipe com um grupocontrole de parto vaginal espontâneo, e constataram que: a mais comum indicação para uso do fórcipe foi a iminente asfixia fetal; o segundo estágio do parto foi mais prolongado e a hospitalização foi mais duradoura com seu uso, e os recém-nascidos apresentaram piores condições pós-parto. Neste, o fórcipe de Simpson-Braun foi indicado quase que exclusivamente para abreviação de período expulsivo e para os partos complicados. Portanto, é natural que a evolução dos neonatos seja pior quando comparada àquela dos neonatos assistidos por parto vaginal espontâneo. Em nosso estudo, observamos que a indicação do fórcipe para 
abreviação do período expulsivo correspondeu a $25 \%$ dos casos, sendo observado um caso de anóxia neonatal grave neste grupo.

Observações isoladas sugerem maior probabilidade de lesões maternas e neonatais nos partos a fórcipe. Entretanto, Hankins et al. ${ }^{13}$ não encontraram evidências de que o fórcipe seja fator de risco para tais lesões e o mesmo se pode concluir deste nosso estudo.

Concluímos que o uso do fórcipe de SimpsonBraun em nossa maternidade mostrou-se seguro, quando comparado ao parto vaginal espontâneo, e que situações de depressão neonatal estão relacionadas a outras variáveis de evolução do parto, que não a aplicação do fórcipe.

\section{ABSTRACT}

Purpose: to compare maternal and neonatal outcomes between spontaneous vaginal and Simpson-Braun forceps deliveries in nulliparous women.

Method: a retrospective study including two groups of nulliparous women, who had vaginal delivery under peridural anesthesia in the Obstetric Unit of the CAISMUNICAMP: the forceps group included 119 women who had Simpson-Braun forceps delivery, and the normal group included 114 women who delivered spontaneously. Neonatal outcomes, such as Apgar score and the evolution in the first days of life, were studied. Data were compared in both groups and, for statistical analysis, $\chi^{2}$ test, Fisher exact, and Student $\mathrm{t}$ tests were used. The differences were considered significant when $p<0.05$.

Results: the indications for Simpson-Braun forceps delivery were maternal-fetal relief ( 90 cases) and abbreviation of the expulsive period (29 cases). In the forceps group there were 8 cases (6.7\%) of vaginal injuries; a similar result was observed in the normal group. The number of hospitalization days for the parturient and the newborns was identical, 2.4 days. The newborns in the two groups had similar Apgar scores inferior to 7 at the first minute (7.5 vs $4.3 \%$ ) and at the fifth minute (1.6 vs $1.7 \%)$. The weight in the two groups had similar results $(3,146$ and 3,016 g). The first days of life did not differ between the groups.

Conclusions: the use of Simpson-Braun forceps was safe, when compared to spontaneous vaginal delivery.

KEYWORDS: Vaginal delivery. Forceps. Maternal death. Neonatal results.

\section{Referências}

1. Neme B. Intervenções durante o parto In: Neme B, editor. Obstetrícia Básica. $2^{a}$ ed. São Paulo: Sarvier; 2000. p.1071-85.

2. Ministério da Saúde. Parto, aborto e puerpério. Assistência humanizada à mulher. Brasília: Ministério da Saúde; 2001.

3. Bofill JA, Rust OA, Schorr SJ, et al. A randomized prospective trial of the obstetric forceps versus the M-cup vacuum extractor. Am J Obstet Gynecol 1996; 175:1325-30.

4. Gardberg M, Ahinko-Hakamaa K, Laakkonen E, Kivela P. Use of obstetric forceps in Finland today: experience at Vaasa Central Hospital. Acta Obstet Gynecol Scand 1999; 78:803-5.

5. Ezenagu LC, Kakaria R, Bofill JA. Sequential use of instruments at operative vaginal delivery: is it safe. Am J Obstet Gynecol 1999; 180:1446-9.

6. Johanson RB, Heycock E, Carter J, Sultan AH, Walklate K, Jones PW. Maternal and child health after assisted vaginal delivery: five-year follow up of a randomised controlled study comparing forceps and ventouse. Br J Obstet Gynecol 1999; 106:544-9.

7. MacArthur C, Glazener CM, Wilson PD, et al. Obstetric practice and faecal incontinence three months after delivery. Br J Obstet Gynecol 2001; 108:678-83.

8. Robinson JN, Norwitt ER, Cohen AP, McElrath TF, Lieberman ES. Episiotomy, opeative vaginal delivery, and significant perinatal trauma in nulliparous women. Am J Obstet Gynecol 1999; 181:1180-4.

9. Wood J, Amos L, Rieger N. Third degree anal sphincter tears: risk factors and outcome. Aust N Z J Obstet Gynaecol 1998; 38:414-7.

10.Towner D, Castro MA, Eby-Wilkens E, Gilbert WM. Effect of mode of delivery in nulliparous women on neonatal intracranial injury. N Engl J Med 1999; 341:1709-14.

11.Carmona F, Martinez-Roman S, Manau D, Cararach $\mathrm{V}$, Iglesias X. Immediate maternal and neonatal effects of low-forceps delivery according to the new criteria of the American College of Obstetricians and Gynecologists compared with spontaneous vaginal delivery in term pregnancies. Am J Obstet Gynecol 1995; 173:55-9.

12.Wydra D, Rogoza A, Szczurowicz A, Olszewski J, Tomczyc P. Forceps delivery: obstetric indications and outcome. Ginekol Pol 1996; 67:341-5.

13.Hankins GD, Leicht T, Vanhook J, Uckan EM. The role of forceps rotation in maternal and neonatal injury. Am J Obstet Gynecol 1999; 180:231-4. 